\title{
Vigilância Sanitária, Participação Social e Cidadania
}

\section{Sanitary Surveillance, Social Participation and Citizenship}

\author{
Ana Maria Caldeira Oliveira \\ Mestre em Saúde Pública. Fiscal Sanitário Municipal da Secretaria \\ Municipal de Saúde de Belo Horizonte. \\ Endereço: Av. Afonso Pena, 2336, térreo, Funcionários, CEP 30130- \\ 007, Belo Horizonte, MG, Brasil. \\ E-mail: anamariacaldeiraœpbh.gov.br \\ Sueli Gandolfi Dallari \\ Livre Docente. Professora Titular do Departamento de Prática de \\ Saúde Pública da Faculdade de Saúde Pública da Universidade \\ de São Paulo. \\ Endereço: Av. Dr. Arnaldo, 715, Sala 102, Subsolo, CEP 01246-904, \\ São Paulo, SP, Brasil. \\ E-mail: sdallariळusp.br
}

\section{Resumo}

O objetivo deste estudo é descrever e analisar as representações sociais dos conselheiros de saúde sobre a temática da vigilância sanitária. A pesquisa qualitativa de representação social foi adotada como metodologia, sendo utilizada a técnica de entrevista baseada em roteiro semiestruturado. Os dados obtidos com a realização das entrevistas foram analisados pela técnica do Discurso do Sujeito Coletivo. Através dos discursos, os conselheiros de saúde demonstraram conhecer a vigilância sanitária e reconhecerem sua importância para as práticas de Saúde Pública. Demonstraram também, estarem aptos a participar do processo de formulação da Política Municipal de Vigilância Sanitária. O estudo conclui que a vigilância sanitária, principalmente na esfera local, precisa se apropriar dos conselhos de saúde como espaços públicos capazes de legitimar e dar transparência às suas ações, discutindo as necessidades da coletividade democraticamente com a sociedade, sendo possível, dessa forma, construir a cidadania ao mesmo tempo em que se assegura o direito à proteção da saúde.

Palavras-chave: Vigilância sanitária; Participação social; Conselhos de saúde. 


\section{Abstract}

The purpose of the study is to describe and analyze the Health Counselors' social representations about the Sanitary Surveillance theme. The social representation qualitative research was the adopted methodology, and the technique of partially structured interviews was used. Data obtained from the interviews were analyzed by means of the Discourse of the Collective Subject. Through the discourses, the Health Counselors demonstrated their knowledge about Sanitary Surveillance and their recognition of its importance for the Public Health practices. In addition, they demonstrated their aptitude for participating in the process of developing the Municipal Sanitary Surveillance Policy. The study concludes that Sanitary Surveillance, especially at the local level, needs to appropriate the cities' Health Councils as public spaces that can legitimate and give transparency to its actions, democratically discussing the needs of the community. Thus, we have the opportunity to build citizenship while ensuring the right to health protection.

Keywords: Sanitary Surveillance; Social Participation; Health Councils.

\section{Introdução}

O debate contemporâneo sobre a participação da sociedade civil em esferas públicas tem indicado inúmeras possibilidades e tendências, com a observação direta da estreita relação entre Estado e sociedade. No Brasil, a ideia do controle social foi ressignificada após o processo de redemocratização política e da promulgação da Constituição Cidadã, passando a ser entendida como participação da sociedade na formulação, acompanhamento e verificação das políticas públicas (Stotz, 2006).

Entre os setores da política social, o setor saúde foi o que mais intensa e precocemente incorporou os mecanismos de participação, destacando-se no país por apresentar a mais sólida estrutura de participação (Côrtes, 2009). A Lei Federal no 8.142/90 regulamentou as instâncias de participação e o controle social no Sistema Único de Saúde (SUS) - os conselhos de saúde e as conferências de saúde (Brasil, 199ob). Os conselhos de saúde em caráter permanente e deliberativo devem atuar na formulação de estratégias e no controle da execução da política de saúde da esfera correspondente. Essa lei estabelece que a representação dos usuários deve ser paritária ao conjunto dos demais segmentos, correspondendo, assim, a 50\% dos representantes. Dessa maneira, a participação da população na formulação e implementação de políticas públicas é fortalecida por uma discriminação positiva (Carvalho, 1995).

A participação da sociedade por meio dos mecanismos institucionalizados dos conselhos está baseada na universalização dos direitos sociais, no alargamento do conceito de cidadania e em uma nova compreensão sobre o papel e o caráter do Estado, entendido como arena de conflitos políticos onde diferentes grupos de interesses disputam espaço e atendimento de suas demandas, a partir de um debate público (Carvalho, 1995; Gohn, 2003).

No campo da vigilância sanitária, a participação e o controle social representam mais um desafio. Valla (2006) destaca que a participação da sociedade na elaboração das políticas de proteção e promoção da saúde precisa constituir-se em um dos elementos de construção da cidadania. Inscrita na Constituição como uma das competências do SUS, a vigilância sanitária é definida na Lei Federal nº 8.080/9o como 
um conjunto de ações capaz de eliminar, diminuir ou prevenir riscos à saúde e de intervir nos problemas sanitários decorrentes do meio ambiente, da produção e circulação de bens e da prestação de serviços de interesse da saúde, abrangendo: I - o controle de bens de consumo que, direta ou indiretamente, se relacionem com a saúde, compreendidas todas as etapas e processos, da produção ao consumo; e II - o controle da prestação de serviços que se relacionam direta ou indiretamente com a saúde (Brasil, 1990a).

Segundo Lucchese (2001, p. 52), uma das principais funções do Estado democrático moderno é proteger e promover a saúde e o bem-estar dos cidadãos. Cabe ao Estado zelar pelos interesses coletivos, intervindo nas atividades de particulares, disciplinando-as quando implicarem em risco à saúde pública. Dessa forma, a instituição do Sistema Nacional de Vigilância Sanitária é consequência da responsabilidade do Estado em assegurar o direito à proteção da saúde e se dá formalmente com a Lei Federal n 9.782/99, que criou a Agência Nacional de Vigilância Sanitária (Anvisa) (Brasil, 1999).

À Anvisa é conferida a natureza de autarquia especial, caracterizada por independência administrativa, estabilidade de seus dirigentes e autonomia financeira, estando vinculada ao Ministério da Saúde através de um Contrato de Gestão. 0 processo de descentralização político-administrativo das ações e serviços de vigilância sanitária foi, então, impulsionado com a criação da agência, embora esse processo tenha acontecido tardiamente em relação aos demais componentes do SUS. A definição de um Sistema Nacional de Vigilância Sanitária, constituído pelo órgão federal, pelos estados e municípios, obedece aos postulados do SUS, entre eles, a diretriz da descentralização, que resulta no deslocamento do poder político e decisório para as esferas subnacionais.

Para legitimar essa função de Estado é necessário que haja transparência, informação e participação social. Entretanto, a Anvisa (2007, p. 48) observa que a participação e o controle social só podem ser exercidos mediante o conhecimento pela sociedade do que é vigilância sanitária e, também, mediante o reconhecimento de sua importância como campo de promoção e proteção da saúde. Apesar de estar presente no cotidiano da população, seja através da utilização de medicamentos, cosméticos, alimentos, ou mesmo dos serviços de saúde, como os hospitais, a vigilância sanitária ainda não é (re)conhecida como parte integrante do SUS. Costa (2004) argumenta que as ações de vigilância sanitária sempre existiram, mas com pouca visibilidade para a população, ou mesmo para os profissionais e gestores da saúde, que se acostumaram a identificar essa área com a atuação policial ou burocrático-cartorial.

O objetivo deste trabalho é descrever e analisar as representações sociais dos conselheiros do Conselho Municipal de Saúde de Belo Horizonte (CMSBH) sobre a vigilância sanitária, visando fomentar a participação desses agentes públicos no ciclo da Política Municipal de Vigilância Sanitária.

\section{Metodologia}

A pesquisa aqui apresentada é um estudo do tipo exploratório-descritivo de natureza qualitativa. Como a pesquisa refere-se ao pensamento e ao conhecimento de uma comunidade sobre determinado tema, a pesquisa qualitativa de representação social se apresenta como o método de escolha.

No período de maio a agosto de 2009, foram entrevistados 35 dos 36 membros efetivos do Conselho Municipal de Saúde de Belo Horizonte, utilizandose a técnica de entrevista baseada em roteiro semiestruturado. Os dados obtidos foram analisados pela técnica do Discurso do Sujeito Coletivo (DSC) (Lefèvre e Lefèvre, 2003), que a partir de estratégia discursiva busca tornar mais clara uma dada representação social.

Para isso, foram identificadas as expressõeschave, que são os trechos selecionados do material verbal que melhor descrevem seu conteúdo, e as ideias centrais, que são formulações sintéticas que descrevem o(s) sentido(s) presente nos conjuntos de respostas de diferentes indivíduos, que têm sentido semelhante ou complementar e, então, construídos os Discursos do Sujeito Coletivo.

Este estudo foi aprovado pelo Comitê de Ética em Pesquisa da Secretaria Municipal de Saúde de Belo Horizonte. 


\section{Resultados e Discussão}

Para se acessar as representações sociais dos conselheiros de saúde utilizamos a seguinte questão aberta: Se você tivesse que explicar para um novo conselheiro, o que é vigilância sanitária, de acordo com as normas e leis, o que você diria?.

Um sujeito coletivo apresentou a ideia central Saúde, bem-estar e qualidade de vida, responsabilizou a vigilância sanitária pela proteção da saúde, através de "um conjunto de normas e estruturas que busca trabalhar para preservar a saúde e o bem-estar, proporcionando uma vida saudável para nossa comunidade, para nossa sociedade". Observa-se a percepção da saúde como direito social necessário à garantia de uma vida digna, com qualidade. Dallari (2003) respalda essa percepção afirmando estar a saúde reconhecida e proclamada como direito fundamental da pessoa humana e ser ela necessidade essencial de todos os indivíduos e de todos os povos.

“Todos nós dependemos da vigilância sanitária... porque ela trabalha em prol da vida". Com essa afirmação, constata-se o que Costa (2008) denomina de apropriação social acerca da importância da vigilância sanitária como ação de saúde. A proteção da saúde e da vida é o campo de atuação prioritário da vigilância sanitária, podendo ser também concebido como espaço de exercício da cidadania e do controle social (Lucchese, 2001), ao trabalhar a saúde como direito universal.

Esse sujeito coletivo entende a vigilância sanitária com "uma norma de viver, de agir e trabalhar, de tornar as pessoas mais informadas no sentido de cuidar melhor das coisas que estão presentes no dia a dia”. A I Conferência Nacional de Vigilância Sanitária (Conavisa, 2001) já havia alertado para a necessidade de se promover ações de informação e comunicação visando à construção da consciência sanitária. Entretanto, o que se constata é que decorridos nove anos da realização dessa conferência, somente agora se iniciou, embora debilmente, a articulação com a população (Teixeira e Costa, 2008). Nesse sentido, Santos, citado por Lucchese (20o6, p. 45), propõe que o conhecimento científico aprenda com o senso comum e transforme-se em saber prático, em informações capazes de tornar compreensível à população o risco envolvido em cada situação, realizando, então, a citada "parceria para o bem-estar da população".

Dessa forma, buscar-se-ia traduzir à população aquilo que o sujeito coletivo de outro grupamento definiu como "Trabalho muito complexo". Segundo Lucchese (2001) algumas características, como a complexidade e a multifocalidade, tornam a vigilância sanitária uma das áreas da saúde pública que requerem grande esforço, tanto para sua compreensão quanto para sua operalização.

Controle de doenças, produtos e serviços foi a ideia central de outro discurso do sujeito coletivo. Essa ideia foi a mais recorrente entre o conjunto de respostas e explica a vigilância sanitária a partir de uma visão ampliada. A vigilância sanitária é "um órgão vigilante em relação às epidemias, devendo vigiar a ocorrência das doenças", e ser capaz também, de dar respostas rápidas às emergências de saúde pública, "igual está chegando agora essa nova gripe, que é uma coisa que tem que estar atento, que tem que ter estratégia para combater". Assim, o sujeito coletivo se referiu à Influenza $\mathrm{A}\left(\mathrm{H}_{1} \mathrm{~N} 1\right)$, chamando a atenção para o processo de globalização dos riscos, o que exige, segundo Dallari (2008), a implementação de um complexo sistema de vigilância sanitária nacional e internacional.

Essa mesma vigilância sanitária "faz a avaliação, a fiscalização, a autorização de circulação e comercialização de produtos”. Trabalha na garantia da qualidade, segurança e eficácia de produtos e serviços de natureza diversa: "alimentos, medicamentos, água destinada ao consumo humano, restaurantes, bares, escolas, instituições de longa permanência, consultórios, farmácias entre outros". Realiza, também, a vigilância de produtos e serviços "que possam trazer risco à saúde da população”. Dessa maneira, observa-se que no imaginário da maioria dos conselheiros de saúde o debate atual acerca da constituição da vigilância em saúde já se resolveu, pelo menos no que diz respeito à integração das ações das vigilâncias - sanitária e epidemiológica.

Existem também, sujeitos coletivos que afirmaram desconhecer a vigilância sanitária. Citando para tanto, duas razões. A primeira seria a ausência da vigilância sanitária no conselho municipal de saúde. Sugeriram, inclusive, a participação da vigilância 
sanitária nas reuniões do conselho municipal. "Que tenha a participação da vigilância sanitária para esclarecer para todos nós o que é vigilância sanitária realmente e como funciona".

Nesse sentido, os participantes da I Conavisa (2001, p. 114) ressaltaram:

A democratização da informação é uma exigência para o exercício pleno dos direitos, devendo ser acionadas diversas estratégias para tornar as ações de vigilância sanitária conhecidas e valorizadas. Reafirmaram que os conselhos de saúde são espaços legítimos para a manifestação dos distintos segmentos sociais sobre questões relacionadas com a saúde e qualidade de vida.

A segunda razão é relacionada à questão da autonomia da vigilância sanitária. "Parece que ela se coloca sempre dentro de uma grande instância chamada agência". Vale observar como a marca Anvisa é emblemática, levando não só os conselheiros, mas a população em geral, a associar a vigilância sanitária municipal à agência e muitas vezes não as distinguir. Para Dallari (2008), a grande autonomia alcançada pela agência põe em risco seu controle democrático e dificulta a identificação de responsabilidades por parte da população e do poder político, embora a mantenha relativamente livre de ingerências políticas e eleitorais. Souza (2007) destaca a tensão entre centralizar e descentralizar existente na criação da Agência Nacional de Vigilância Sanitária. Para se ajustar ao modelo de agência reguladora, consequência do movimento de desregulação, o poder foi ampliado, legitimado e centralizado. Ao mesmo tempo, compete à agência a tarefa de coordenar o Sistema Nacional de Vigilância Sanitária, sendo necessário viabilizar a diretriz constitucional da descentralização, instituindo o processo de descentralização das ações e serviços aos entes federados.

A ideia central Parte integrante do SUS revela um importante esclarecimento feito por esse sujeito coletivo. "Todos nós somos usuários do SUS em função da vigilância sanitária”. Com essa afirmação, os conselheiros demonstraram perceber o caráter universal que a vigilância sanitária traz agregado à suas ações e serviços. Reconheceram a vigilância sanitária como "parte importante do SUS" e, por- tanto, como parte integrante do Sistema Único de Saúde. Sendo parte integrante do SUS a vigilância sanitária "pode intervir com uma ação conjunta da epidemiologia e da saúde do trabalhador, que hoje é importante a vigilância assumir". Dessa forma, a visão integradora, que articula as práticas da vigilância sanitária com as da vigilância epidemiológica e de saúde do trabalhador, antecipa a Portaria/MS 3252 de dezembro de 2009, que aprova as diretrizes para a execução das ações de vigilância em saúde. Essas diretrizes dizem respeito exatamente à integração das ações das vigilâncias - sanitária, epidemiológica e ambiental, com ações voltadas para a saúde do trabalhador (Brasil, 2009).

Por sua vez, outro sujeito coletivo com a ideia central Modelo de promoção e prevenção enfatizou a importância do papel da vigilância sanitária na prevenção e promoção da saúde. “[...] Nós temos que lembrar que você tem que trabalhar a saúde para não adoecer. A vigilância sanitária é uma das almas desse processo de inversão do modelo menos curativo... para mais promoção e prevenção". Lucchese (2006) afirma que a atividade de vigilância sanitária, além de fazer parte das competências do SUS, possui caráter prioritário por sua natureza essencialmente preventiva. Esse mesmo sujeito coletivo observou: "a vigilância sanitária pressupõe uma proatividade, um olhar atento, que vai além da inspeção periódica de concessão de alvará ou autorização de funcionamento". Com efeito, é preciso avançar. A vigilância sanitária é, sem dúvida, muito maior do que sua atividade de execução, de verificação da conformidade e da não conformidade. É preciso priorizar a atividade de vigilância propriamente dita e, em conjunto com os demais componentes do SUS, trabalhar no desenvolvimento integral dessa atividade estratégica para que se possa assegurar qualidade de vida e bem-estar aos cidadãos.

É nesse sentido que o Ministério da Saúde, ao editar a Portaria 3252/o9, buscou reorganizar suas práticas, ajustando o modelo de atenção para proporcionar uma análise permanente da situação de saúde da população. Para tanto, constitui a vigilância em saúde com ações de promoção da saúde, vigilância, proteção, prevenção e controle das doenças e agravos à saúde (Brasil, 2009, arts. $1^{\circ}$ e $\left.2^{\circ}\right)$. Nesse contexto, se faz urgente que a vigilância sanitária acompanhe 
essa nova reforma incremental do SUS e reconfigure seu objeto, qualificando suas ações. Costa (2008, p. 87) propõe "que as ações sejam direcionadas a riscos, danos, necessidades sanitárias e determinantes do processo saúde-doença-cuidado-qualidade de vida, compondo o campo da proteção e promoção da saúde".

O sujeito coletivo com a segunda maior incidência de ideias centrais explicou a vigilância sanitária através de seu lado mais visível, o Órgão fiscalizador. A Constituição Federal de 1988, no artigo 197, estabelece como de relevância pública as ações e serviços de saúde, o que pressupõe do Poder Público o exercício da atividade regulatória, através da fiscalização, controle e regulamentação dessa atividade. Esse sujeito coletivo observou um "Poder Público vigilante, com aquela proposta de fiscalizar, de controlar". Silva (1994, p. 707) comenta que, se a Constituição atribui ao Poder Público o controle das ações e serviços de saúde, significa que sobre tais ações e serviços tem ele integral poder de dominação, que é o sentido do termo controle, sobretudo quando aparece ao lado da palavra fiscalização. Nesse sentido, a vigilância sanitária pode ser definida como campo de intervenção do Estado, com a capacidade, devido às suas funções e instrumentos, de trabalhar de modo a adequar o sistema produtivo de bens e serviços de interesse sanitário às demandas sociais de saúde e as necessidades do sistema de saúde (Lucchese, 2001).

Então, eu verifico que ali tá faltando a vigilância sanitária. Não tá tendo o rigor, seguindo as regras que tem que seguir. Aí a gente procura a vigilância sanitária porque ela é o órgão que tem que fazer o trabalho, né? [...] ela vai verificar, vai registrar, vai entrar com multa... alguma coisa ela vai fazer. (Conselheiro do segmento usuário)

Nas palavras de Costa (2008), o poder de polícia é fundamentado no princípio da predominância do interesse público sobre o particular, sendo essencial para a imposição de normas e padrões de comportamento. Dessa forma, a vigilância sanitária utiliza instrumental legal, que é pré-requisito para sua atuação. A lei deve expressar o sistema jurídico definido na Constituição, afirmando assim sua legitimidade. As normas técnicas utilizadas integram um sistema de normas sanitárias que representam os conhecimentos científicos e tecnológicos e, também, os interesses da saúde pública. Ainda segundo a autora, a competência para impor condicionamentos se faz acompanhar da necessária competência para fiscalizar seu cumprimento, tarefa intransferível da vigilância sanitária e necessária para a proteção e defesa da saúde. Corresponde exatamente ao pensamento desse sujeito coletivo ao expressar "a atividade de vigilância sanitária decorre dessa autoridade, desse cuidado, dessa responsabilidade com a saúde pública em todas as acepções". Esse sujeito coletivo ainda percebeu que "infelizmente, a vigilância não tem condições de fazer uma fiscalização como deve ser feita". O reconhecimento dos conselheiros de saúde sobre a insuficiência da ação fiscalizadora coaduna com o pensamento expresso por Lucchese (2001) de que o sistema de vigilância sanitária precisa contar com o apoio da sociedade na vigilância dos riscos sob sua responsabilidade.

\section{Considerações Finais}

Ao buscarmos desvelar as representações sociais dos Conselheiros de Saúde de Belo Horizonte sobre o tema vigilância sanitária, detectamos a existência de algumas matrizes discursivas predominantes. A vigilância sanitária é entendida como órgão fiscalizador pertencente ao Estado, que realiza o controle das doenças, produtos e serviços, colaborando na garantia de sua qualidade. Tem a responsabilidade de preservar a saúde e o bem-estar, proporcionando uma vida saudável para os cidadãos. Os conselheiros percebem, também, a natureza preventiva da vigilância sanitária e o seu pertencimento ao SUS.

Os conselheiros de saúde, através de suas representações sociais, demonstraram conhecer a vigilância sanitária e reconhecerem sua importância para as práticas de Saúde Pública, inclusive, responsabilizando-a pela proteção da saúde da população. Além disso, os conselheiros revelaram interesse em conhecer, discutir e acompanhar todo o trabalho desenvolvido pela vigilância sanitária. Fica evidente a necessidade de se fomentar um permanente debate entre os segmentos da sociedade com a vigilância sanitária. Nesse contexto, os conselheiros demonstraram, também, estarem aptos 
a participarem, através do exercício de cidadania e do controle social, do processo de formulação, acompanhamento e verificação da Política Pública Municipal de Vigilância Sanitária.

Por fim, concluímos que a vigilância sanitária, principalmente na esfera local, precisa se apropriar dos conselhos de saúde como espaços públicos capazes de legitimar e dar transparência às suas ações, discutindo as necessidades da coletividade democraticamente com a sociedade, sendo possível, dessa forma, construir a cidadania ao mesmo tempo em que se assegura o direito à proteção da saúde.

\section{Referências}

AGÊNCIA NACIONAL DE VIGILÂNCIA SANITÁRIA (ANVISA). Plano diretor de vigilância sanitária. Brasília, DF: Agência Nacional de Vigilância Sanitária, 2007.

BRASIL. Constituição (1988). Constituição da República Federativa do Brasil. Brasília, DF: Senado Federal, 1988.

BRASIL. Lei n. 8.080, de 19 de setembro de 1990. Dispõe sobre as condições para a promoção, proteção e recuperação da saúde, a organização e o funcionamento dos serviços correspondentes e dá outras providências. Diário Oficial da União, Brasília, DF, 20 de set. 199o. p. 18055.

BRASIL. Lei n. 8.142, de 28 de dezembro de 1990. Dispõe sobre as transferências intergovernamentais de recursos financeiros na área da saúde. Diário Oficial da União, Brasília, DF, 31 de dez. 1990.

BRASIL. Lei nº 9.782, de 26 de janeiro de 1999. Define o Sistema Nacional de Vigilância Sanitária, cria a Agência Nacional de Vigilância Sanitária, e dá outras providências. Diário Oficial da União, Brasília, DF, 27, jan. 1999.

BRASIL. Ministério da Saúde. Portaria GM/ MS n. 3.252, de 22 de dezembro de 2009. Aprova as diretrizes para execução e financiamento das ações de vigilância em saúde pela União, estados, Distrito Federal e municípios e dá outras providências. Diário Oficia l da União, Brasília, DF, 23 dez. 2009. p. 12.
CARVALHO, A. I. Conselhos de saúde no Brasil. Rio de Janeiro: IBAM/FASE, 1995. CONFERÊNCIA NACIONAL DE VIGILÂNCIA SANITÁRIA, 1, 2001, Brasília. Conferência Nacional de Vigilância Sanitária: relatório final. Brasília: Agência Nacional de Vigilância Sanitária, 2001.

CÔRTES, S. M. V. Conselhos e conferências de saúde: papel institucional e mudança nas relações entre Estado e sociedade. In: FLEURY, S.; LOBATO, L. V. C. (Org.). Participação, democracia e saúde. Rio de Janeiro: Cebes, 2009. p. 102-127.

COSTA, E. A. Vigilância sanitária: proteção e defesa da saúde. 2. ed. São Paulo: Sobravime, 2004 .

COSTA, E. A. O trabalhador de vigilância sanitária e a construção de uma nova vigilância: fiscal ou profissional de saúde? In: COSTA, E. A. Vigilância sanitária: desvendando o enigma. Salvador: Edufba, 2008. p. 77-90.

DALLARI, D. A. Ética sanitária. In: MINISTÉRIO DA SAÚDE. Direito sanitário e saúde pública. Brasília, DF, 2003.v. 1, p. 65-86.

DALLARI, S. G. Vigilância sanitária: responsabilidade pública na proteção e promoção da saúde. In: COSTA E. A. (Org.). Vigilância sanitária: desvendando o enigma. Salvador: Edufba, 2008. p. 45-52.

GOHN, M. G. Conselhos gestores e participação sócio-política. São Paulo: Cortez, 2003.

LEFÈVRE, F.; LEFÈVRE, A. M. C. O discurso do sujeito coletivo: um novo enfoque em pesquisa qualitativa: desdobramentos. Caxias do Sul: EDUCS, 2003.

LUCCHESE, G. Globalização e regulação sanitária: os rumos da vigilância sanitária no Brasil. Rio de Janeiro, 2001. Tese (Doutorado em Saúde Pública) - Escola Nacional de Saúde Pública, Fundação Oswaldo Cruz, Rio de Janeiro, 2001.

LUCCHESE, G. A vigilância sanitária no Sistema Único de Saúde. In: DE SETA, M. H.; PEPE, V. L. E.; OLIVEIRA, G. O. (Org.). Gestão e vigilância sanitária: modos atuais do pensar e fazer. Rio de Janeiro: Fiocruz, 2006. p. 33-47. 
SOUZA, A. M. A. F. Vigilância sanitária na saúde

pública brasileira e sua aproximação com o caso mexicano: proteger, vigiar e regular. 2007. Tese (Doutorado em Saúde Pública) - Faculdade de Saúde Pública, Universidade de São Paulo, São Paulo, 2007.

SILVA, J. A. Curso de direito constitucional positivo. 9. ed. São Paulo: Malheiros, 1994.

STOTZ, E. N. Trajetória, limites e desafios do controle social do SUS. Saúde debate, Rio de Janeiro, v. 3o, n. 73/74, p. 149-16o, maio/dez. 2006.

TEIXEIRA, C. F.; COSTA, E. A. Vigilância da saúde e vigilância sanitária: concepções, estratégias e práticas. In: COSTA, E. A. (Org.). Vigilância sanitária: desvendando o enigma. Salvador: Edufba, 2008. p. 149-90.

VALLA, V. V. Controle social ou controle público. In: DE SETA, M. H.; PEPE, V. L. E.; OLIVEIRA, G. O. (Org.). Gestão e vigilância sanitária: modos atuais do pensar e fazer. Rio de Janeiro: Fiocruz, 2006. p. 49-6o. 